Research paper

\title{
Innovations in diagnosis and post-therapeutic monitoring of Chagas disease: Simultaneous flow cytometric detection of IgG1 antibodies anti-live amastigote, anti-live trypomastigote, and anti-fixed epimastigote forms of Trypanosoma cruzi
}

\author{
Glaucia Diniz Alessio ${ }^{a}$, Denise Fonseca Côrtes ${ }^{a}$, Girley Francisco Machado de Assis ${ }^{a}$, \\ Policarpo Ademar Sales Júnior ${ }^{c}$, Eloisa Amália Vieira Ferro ${ }^{\mathrm{d}}$, Lis Ribeiro do Valle Antonelli ${ }^{\mathrm{e}}$, \\ Andréa Teixeira-Carvalho ${ }^{\mathrm{b}}$, Olindo Assis Martins-Filho ${ }^{\mathrm{b}, *}$, Marta de Lana ${ }^{\mathrm{a}}$ \\ a Laboratório de Doença de Chagas, Núcleo de Pesquisas em Ciências Biológicas (NUPEB), Instituto de Ciências Exatas e Biológicas (ICEB), \\ Universidade Federal de Ouro Preto (UFOP), Ouro Preto, MG, Brasil \\ b Laboratório de Biomarcadores de Diagnóstico e Monitoração, Centro de Pesquisas René Rachou (CPqRR-FIOCRUZ/MG), Belo Horizonte, MG, Brasil \\ c Laboratório de Parasitologia Celular e Molecular, Centro de Pesquisas René Rachou (CPqRR-FIOCRUZ/MG), Belo Horizonte, MG, Brasil \\ d Universidade Federal de Uberlândia, Instituto de Ciências Biomédicas, Departamento de Morfologia, Uberlândia, MG, Brasil \\ e Laboratório de Imunopatologia, Centro de Pesquisas René Rachou (CPqRR-FIOCRUZ/MG), Belo Horizonte, MG, Brasil
}

\section{A R T I C L E I N F O}

\section{Article history:}

Received 16 April 2014

Received in revised form 23 June 2014

Accepted 15 July 2014

Available online $\mathrm{xxxx}$

\section{Keywords}

Chagas disease

Flow cytometry

Serology

Diagnosis

Post-therapeutic monitoring

\begin{abstract}
A B S T R A C T
This study developed a remarkable methodological innovation (FC-ATE) which enables simultaneous detection of antibodies specific to the three evolutive forms of Trypanosoma cruzi: live amastigote (AMA), live trypomastigote (TRYPO), and fixed epimastigote (EPI) using a differential fluorescence staining as low (AMA), intermediate (TRYPO), and high (EPI). An outstanding performance $(100 \%)$ was observed in the discrimination of the chagasic $(\mathrm{CH})$ and non-chagasic ( $\mathrm{NCH}$ ) patients. In the applicability of FC-ATE in the diagnosis of Chagas disease, $100 \%$ of the $\mathrm{CH}$ samples presented positivity in the percentage of positive fluorescent parasites (PPFP) for all the three forms of T. cruzi. Moreover, 94\% of the samples of $\mathrm{NCH}$ presented negative values of PPFP with AMA and TRYPO, and $88 \%$ with EPI. Samples from the NCH group with falsepositive results were those belonging to the leishmaniasis patients. Considering the applicability of this technique in post-therapeutic monitoring of Chagas disease, $100 \%$ of non-treated (NT) and treated non-cured (TNC) samples were positive with the three T. cruzi evolutive forms, while a percentage of $100 \%$ from samples of the treated cured (TC) patients were negative with AMA, $93 \%$ with TRYPO and 96\% with EPI. The comparison between FC-ATE and two other flow cytometric tests using the same samples of patients NT, TNC and TC showed that the three techniques presented different reactivities, although categorical correlation between the methodologies was observed. Taken together, the results obtained with the novel FC-ATE method have shown an outstanding performance in the diagnosis and post-therapeutic monitoring of Chagas disease.
\end{abstract}

(c) 2014 Elsevier B.V. All rights reserved.

Abbreviations: CoML, non-conventional serology complemented mediated lysis; FC-ALTA, flow cytometric analysis of anti-live trypomastigote antibodies; FCAFEA, flow cytometric analysis of anti-fixed epimastigote antibodies; FC-ATE, flow cytometric analysis of anti-live trypomastigote and anti-fixed epimastigote antibodies.

* Corresponding author at: Laboratório de Biomarcadores de Diagnóstico e Monitoração, Centro de Pesquisas René Rachou, Avenida Augusto de Lima 1715, Barro Preto, Belo Horizonte, Minas Gerais, 30.190-002, Brazil. Tel.: +55 313349 7764; fax: +55 3132953115.

E-mail address: oamfilho@cpqrr.fiocruz.br (O.A. Martins-Filho). 


\section{Introduction}

Chagas disease, one of the most hazardous infectious diseases, has as etiological agent the protozoan parasite Trypanosoma cruzi, order Kinetoplastida, family Trypanosomatidae (Chagas, 1909). Currently, Chagas disease constitutes a major public health problem in Latin America, and approximately 10 million people distributed in 21 countries in Latin America are infected with T. cruzi, with an annual incidence of 40,000 new cases per year (Moncayo and Silveira, 2009; WHO, 2010). In the past decades, this infection has been increasingly found in non-endemic countries of several continents, mainly due to increasing population mobility (Schmunis, 2007) in addition to the mechanisms of infection by blood transfusion, vertical transmission and transplants.

The life cycle of $T$. cruzi includes three main morphological stages in two phylogenetically diverse hosts: the arthropod vector, with the replicative epimastigote (EPI) and nonreplicative metacyclic trypomastigote (TRYPO) forms, and the mammalian host, with the replicative amastigote (AMA) and non-replicative bloodstream trypomastigote (TRYPO) forms (Brener, 1973; Dias, 2000).

Clinically, Chagas disease has two successive phases: the acute phase, which lasts for 1 to 4 months and is characterized by patent parasitemia and the chronic phase, which is a lifelong phase with subpatent parasitemia and scarce tissue parasitism (Dias, 1995; Rassi et al., 2010). During the acute phase, the diagnosis is carried out by the direct detection of circulating parasites. However, during the chronic phase, when the parasitemia is low, the diagnosis is performed mainly by serological methods (Sánchez et al., 2008). Serological tests currently used for diagnosis in the chronic phase are enzymelinked immunosorbent assay (ELISA), indirect immunofluorescence (IIF), and indirect hemagglutination (IHA) (Luquetti and Rassi, 2000).

Treatment for Chagas disease is recommended for all people diagnosed with an acute infection, congenital infection, for those with suppressed immune systems, and for all children with chronic infection (Consenso Brasileiro em Doença de Chagas, 2005). Adults with chronic infection may also benefit from treatment (Viotti et al., 1994; Fabbro et al., 2000; Lana et al., 2009; Machado-de-Assis et al., 2012). For post-therapeutic monitoring of Chagas disease in the chronic phase, several laboratorial methods are available, including parasitological (xenodiagnostic and hemoculture) (Chiari et al., 1989; Schenone et al., 2000), molecular (polymerase chain reactionPCR) (Gomes et al., 1998; Britto, 2009), conventional serology (ELISA, IIF, IHA) (Cançado, 2002; Coura and Castro, 2002), and non-conventional serology (complemented mediated lysisCoML, flow cytometric analysis of anti-live trypomastigote antibodies-FC-ALTA, and flow cytometric analysis of anti-fixed epimastigote antibodies-FC-AFEA) (Krettli et al., 1982; Galvão et al., 1993; Martins-Filho et al., 1995; Vitelli-Avelar et al., 2007). Despite the broad range of assays, several methodological limitations, especially in sensitivity and specificity, still remain (Portela-Lindoso and Shikanai-Yasuda, 2003; Gomes et al., 2009).

Aiming to overcome such limitations, the development of new serological tests for Chagas disease should consider the two groups of anti-T. cruzi antibodies, lytic antibodies (LA), and conventional serology antibodies (CSA) (Krettli and Brener,
1982). Lytic antibodies are associated with active ongoing T. cruzi infection and can bind to live trypomastigotes. They are usually detected by CoML or flow cytometry (Krettli and Brener, 1976, 1982; Martins-Filho et al., 1995). CSA are mainly detected by routine methods as ELISA, IIF and IHA or flow cytometry, using soluble and/or fixed antigenic preparations (Luquetti and Rassi, 2000; Vitelli-Avelar et al., 2007).

In this context, several studies have evaluated the reactivity of LA and CSA by flow cytometry in both diagnosis and treatment monitoring (Martins-Filho et al., 1995; Cordeiro et al., 2001; Martins-Filho et al., 2002; Vitelli-Avelar et al., 2007; Matos et al., 2011; Wendling et al., 2011). FC-ALTA, using live trypomastigotes as antigens, assesses LA, and FC-AFEA, using fixed epimastigotes as antigens, assesses CSA. Both have shown high-quality performance in the diagnosis and posttherapeutic monitoring of Chagas disease (Martins-Filho et al., 1995, 2002; Cordeiro et al., 2001; Vitelli-Avelar et al., 2007; Matos et al., 2011; Wendling et al., 2011).

Some studies have evaluated the reactivity of anti-amastigote also in serological tests for Chagas disease. These specific antibodies against AMA exhibited higher reactivity for the serological diagnosis of Chagas disease than antibodies to EPI (Cerisola et al., 1971; Araujo and Guptill, 1984; Primavera et al., 1990; Matsumoto et al., 1993). Matsumoto et al. (1993) showed that IgG anti-AMA was more reactive than IgG anti-EPI. Still, when AMA was used as antigen a lower percentage of crossreactivity was observed. Previous studies with amastigotes have focused on the development of DNA vaccines against infection by T. cruzi using SSP4 glycoprotein found in the plasma membrane of these forms (Ramos-Ligonio et al., 2004; Arce-Fonseca et al., 2011; Flores-García et al., 2011). However, there are few studies using amastigotes as antigens in serological tests for Chagas disease.

Our current study has proposed to establish a more sensitive and specific methodological innovation by optimizing the performance of flow cytometry anti-T. cruzi serology. The main innovation was the use of a more complex antigen preparation, consisted by a mixture of alive AMA and TRYPO along with fixed EPI. Using this approach, it was possible to combine sensitivity, by the EPI antigen recognition, and specificity, by the AMA and TRYPO antigen recognition. The method namely flow cytometric analysis of anti-live amastigote, anti-live trypomastigote, and anti-fixed epimastigote IgG1 antibodies (FC-ATE) has shown a great performance for the diagnosis and post-therapeutic monitoring of Chagas disease.

\section{Population, materials and methods}

\subsection{Study population}

The inclusion of all individuals in this investigation had the approval of the Ethics Committee at René Rachou Research Center, Oswaldo Cruz Foundation-FIOCRUZ, Minas Gerais, Brazil. All human subjects sign the informed consent form. Three groups of patients were used to achieve the proposed aims.

\subsubsection{Group 1}

To standardize the FC-ATE assay, 53 serum samples were used: 22 from the chagasic patients $\left(\mathrm{CH}_{1}\right)$, including 12 nontreated (NT), 10 treated non-cured (TNC), and 31 from the 
non-chagasic patients $\left(\mathrm{NCH}_{1}\right)$, including 10 blood donor (BD), and 21 treated cured (TC) individuals.

The NT, TNC and TC patients were assisted by a clinical physician at the Faculty of Medicine, Federal University of Goiás, Goiânia, Brazil. The ages of the patients ranged from 6 months to 66 years. Chagas disease diagnosis was established in all patients by positivity in the xenodiagnosis, and three serological tests, including IHA, IIF and ELISA.

The TNC and TC patients were previously submitted to etiological treatment with Benznidazole $(5 \mathrm{mg} / \mathrm{kg}$ of body weight/day for 60 days) followed by clinical, parasitological (xenodiagnosis as described by Cerisola et al., 1971), and serological follow-up analyses ranging 3 to 26 years after etiological treatment. Based on laboratorial analyses (parasitological and serological tests), patients were categorized as TNC $(\mathrm{n}=10)$ and TC $(\mathrm{n}=21)$. The TNC patients presented consistently positive results for both xenodiagnosis and serological tests (IHA, IIF and ELISA) during the follow-up. Patients considered as TC presented persistent negative results in xenodiagnosis and conventional serological tests (IHA, IIF and ELISA).

The BD individuals $(n=10)$ were volunteers who attended the Felício Rocho Hospital, Belo Horizonte, Minas Gerais, Brazil.

\subsubsection{Group 2}

To evaluate the applicability of FC-ATE in the diagnosis of Chagas disease, a group of 63 serum samples were analyzed, and consisted of 12 non-treated chagasic $\left(\mathrm{CH}_{2}\right)$, and 51 nonchagasic $\left(\mathrm{NCH}_{2}\right)$ patients including $10 \mathrm{BD}, 21 \mathrm{TC}, 10$ patients with visceral leishmaniasis (VL), and 10 patients with American cutaneous leishmaniasis (ACL). $\mathrm{CH}_{2}, \mathrm{BD}$ and $\mathrm{TC}$ samples were the same as those used in Group 1. Samples from the VL and ACL patients were provided by the biorepository at the Laboratory of Biomarkers for Diagnosis and Monitoring, René Rachou Research Center, Oswaldo Cruz Foundation, FIOCRUZ, Minas Gerais, Brazil.

\subsubsection{Group 3}

To evaluate the applicability of FC-ATE in the posttherapeutic monitoring of Chagas disease, 69 serum samples were analyzed, and consisted of 12 NT, 29 TNC, and 28 TC samples. All serum samples were the same as those used in Group 1. Moreover, these samples were used to analyze the performance of FC-ATE in comparison with two nonconventional serological flow cytometric assays developed by our group (FC-ALTA and FC-AFEA).

The evaluated TC $(n=7)$ and TNC $(n=16)$ patients were from the Berilo municipality, Jequitinhonha Valley, Minas Gerais, Brazil, a vector-controlled area of Chagas disease. These patients were treated with Benznidazole at least 10 years ago at a dosage of $5 \mathrm{mg} / \mathrm{kg}$ for 60 days. Before treatment, the Chagas disease diagnosis was confirmed by two conventional serological tests. Patients were categorized as TNC when results were positive for hemoculture and conventional serology tests (IHA, IIF and ELISA), and TC when patients presented persistent negative results in hemoculture and conventional serology tests (IHA, IIF and ELISA) (Machado-de-Assis et al., 2012). Three TNC patients were volunteers who attended the Ambulatory of Chagas Disease at Clinics Hospital, Federal University of Minas Gerais, Belo Horizonte, Brazil, in the period from 1997 to 2005.

\subsection{Parasite preparations}

\subsubsection{Trypomastigotes and amastigotes}

For the assays, $5 \times 10^{5}$ L929 cells were seeded in tissue culture flasks (Falcon 25 or $75 \mathrm{~cm}^{2}$ ) with $10 \mathrm{ml}$ of RPMI medium 1640 (GIBCO), phenol red with 10\% FBS, and $2 \mathrm{mM}$ glutamine before incubation at $37^{\circ} \mathrm{C}$ in a humidified air with $5 \%$ $\mathrm{CO}_{2}$. After 2 or 3 days, the monolayer was infected with $5 \times 10^{6}$ TRYPO of $T$. cruzi CL strain (Brener, 1965) obtained from experimentally infected mice. Cultures were maintained in RPMI $10 \%$ of FBS at $33{ }^{\circ} \mathrm{C}$ in $5 \% \mathrm{CO}_{2}$ at $95 \%$ humidity (Bertelli et al., 1977). After 5 days, TRYPO were harvested from the supernatant. To obtain AMA forms, cultures were maintained for 10 days at $33{ }^{\circ} \mathrm{C}$ in $5 \% \mathrm{CO}_{2}$ at $95 \%$ humidity in RPMI medium with $10 \%$ of FBS. However, it is known that pure cultures of AMA or TRYPO are difficult to obtain, so that both forms were present in the same culture, but the culture denominated TRYPO had a greater proportion of trypomastigotes and the culture denominated AMA had a large amount of amastigotes. The culture denominated TRYPO and AMA were collected separately in $50 \mathrm{ml}$ falcon tubes. Cell debris were removed from the cultures by differential centrifugation at $400 \mathrm{rpm}$ for $10 \mathrm{~min}$ at room temperature. The supernatants were centrifuged at $900 \times \mathrm{g}$ for $10 \mathrm{~min}$ at $4^{\circ} \mathrm{C}$. The pellets were washed in PBS, pH 7.2, supplemented with $10 \%$ of FBS. In the TRYPO suspension the trypomastigotes were adjusted to $1 \times 10^{7} / \mathrm{ml}$ and in the AMA suspension the amastigotes were adjusted to $1 \times 10^{7} / \mathrm{ml}$. Afterwards, the preparation containing both parasites was stained and used to flow cytometric immunofluorescence assay.

\subsubsection{Epimastigotes}

T. cruzi epimastigotes of CL strain were grown in axenic culture in liver infusion tryptose medium - LIT (Camargo, 1964) and maintained at $28^{\circ} \mathrm{C}$. Each culture batch was started with an initial inoculum of $1 \times 10^{7}$ parasites $/ \mathrm{ml}$ using $3 \mathrm{ml}$ of LIT. The epimastigote forms were homogenized daily for aeration, and every seven days the cultures were subculture by serial passages in vitro. Thus, EPI forms were harvested during the log phase of growth. Parasites were washed twice with $3 \%$ FBS-PBS $\left(900 \times g\right.$ for $10 \mathrm{~min}$ at $18{ }^{\circ} \mathrm{C}$ ), immediately resuspended in equal volume of $3 \%$ FBS-PBS and FACS fix solution (10.0 $\mathrm{g}$ of paraformaldehyde, $10.2 \mathrm{~g}$ of sodium cacodylate and $6.65 \mathrm{~g}$ of sodium chloride/l, pH 7.2; Sigma Chemical) and maintained at $4{ }^{\circ} \mathrm{C}$ for $24 \mathrm{~h}$. Following, the parasites were centrifuged at $900 \times g$ for $7 \mathrm{~min}$ at $4{ }^{\circ} \mathrm{C}$, resuspended in $1 \mathrm{ml}$ of PBS and stored at $4{ }^{\circ} \mathrm{C}$ until use. This preparation is stable in this condition for up to one year. The suspension of parasites was adjusted to $1 \times 10^{7} / \mathrm{ml}$.

\subsection{Preparation of T. cruzi and cell sorting}

A culture containing both trypomastigote and amastigote forms was used for the cell sorting. The culture was washed in PBS, pH 7.2, supplemented with $10 \%$ of FBS and stained with $200 \mu \mathrm{g} / \mathrm{ml}$ of fluorescein isothiocyanate (FITC) at $37^{\circ} \mathrm{C}$ for $30 \mathrm{~min}$. The parasites were washed three times with PBS $10 \%$ FBS $\left(900 \times g\right.$ for $10 \mathrm{~min}$ at $\left.18{ }^{\circ} \mathrm{C}\right)$. The culture was incubated at $37{ }^{\circ} \mathrm{C}$ for $60 \mathrm{~min}$. Parasite staining was evaluated by flow cytometry scanning. Parasites were centrifuged at $900 \times \mathrm{g}$ for $10 \mathrm{~min}$ at $4{ }^{\circ} \mathrm{C}$, resuspended in $2 \mathrm{ml}$ of FACS 
A
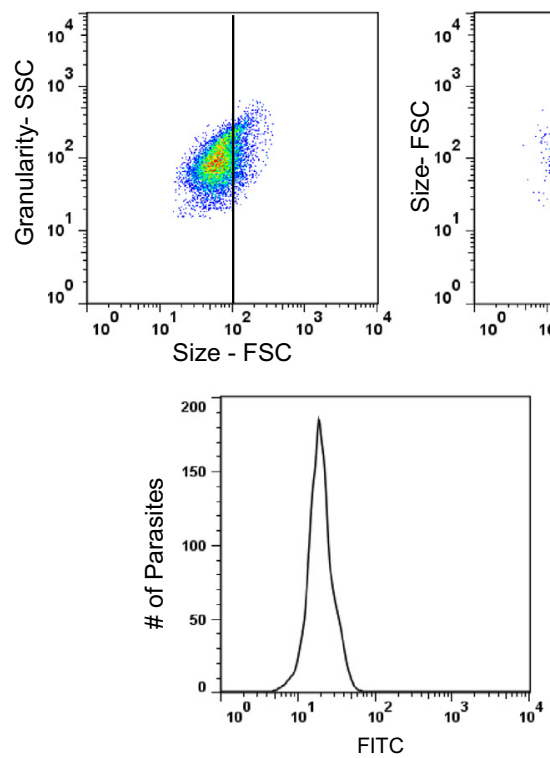

AMA + TRYPO
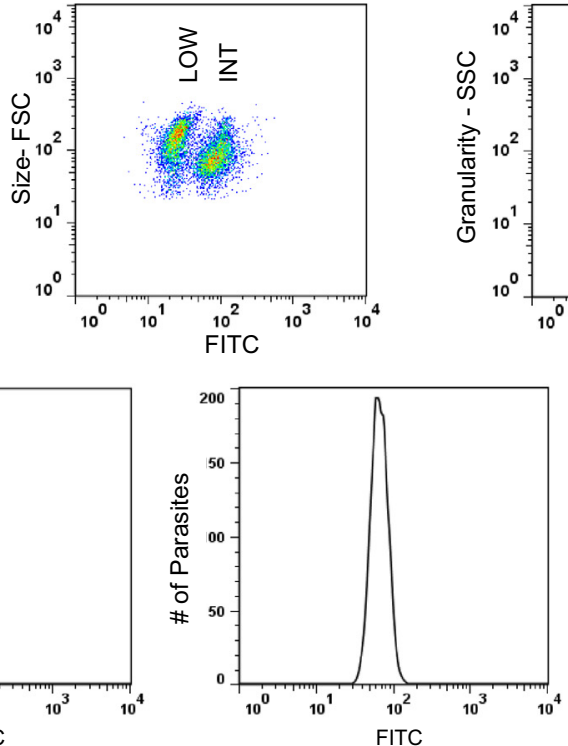

EPI
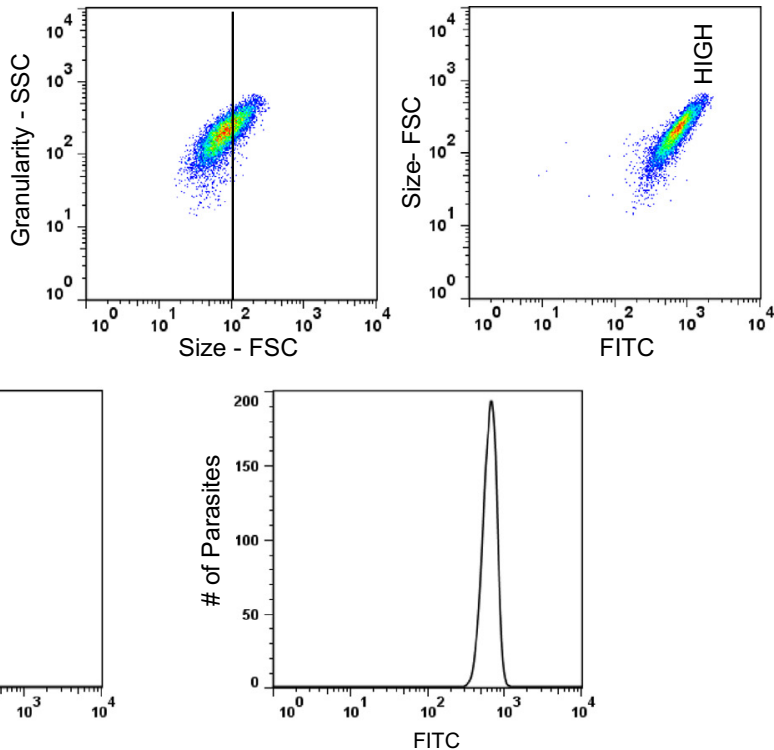

B
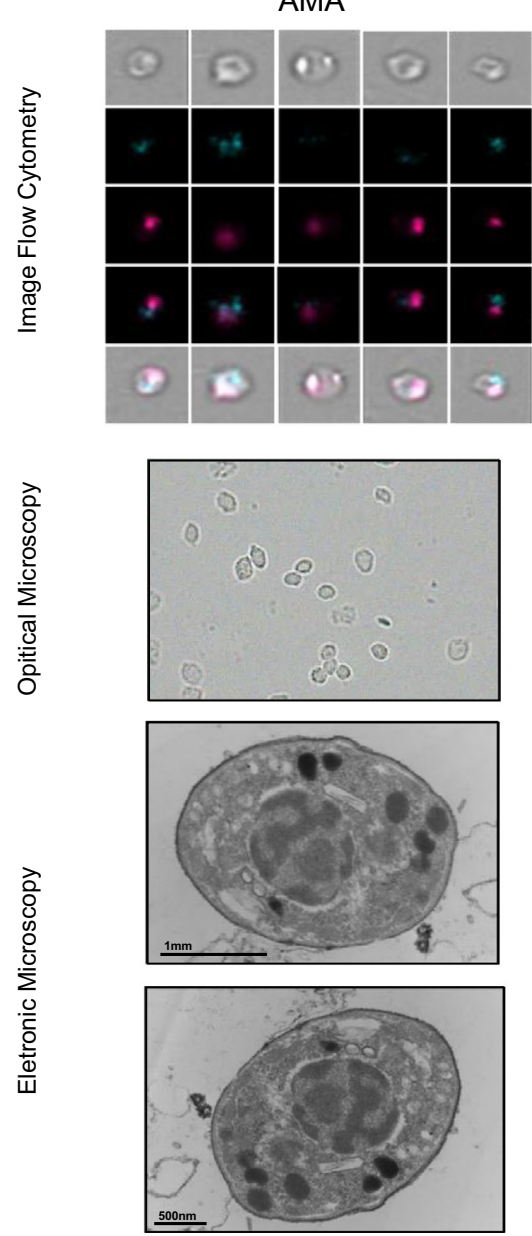

TRYPO
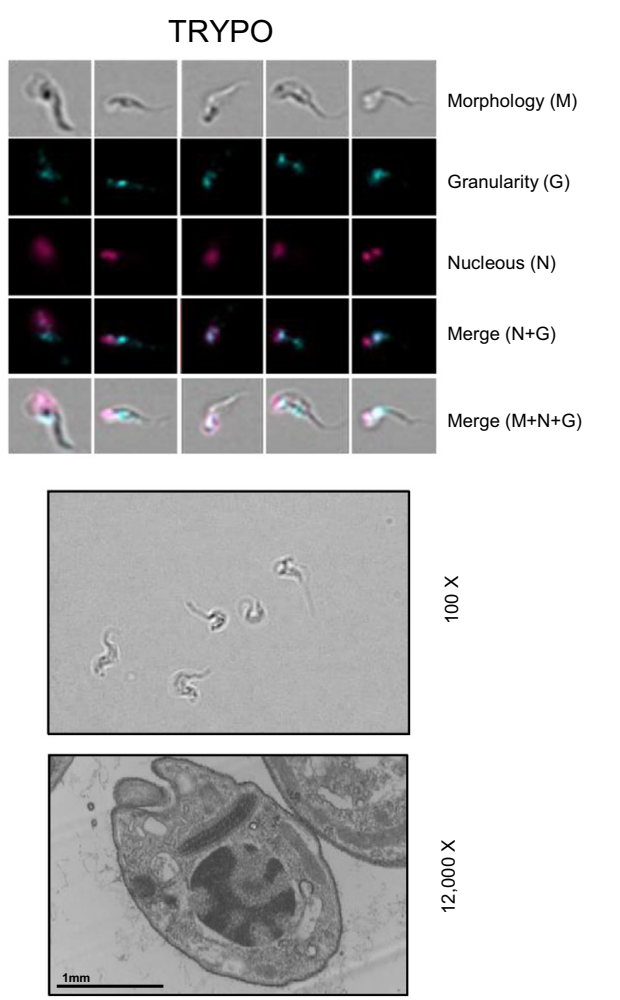

$\times$
응
ำ

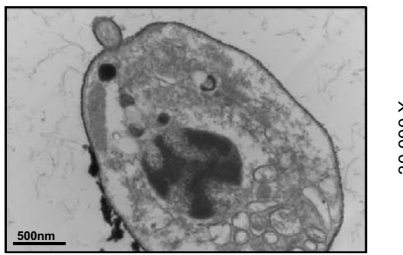

$\stackrel{\times}{\circ}$

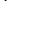

$\times$
$\stackrel{ }{\circ}$
$\stackrel{+}{-}$

悹 
fixing solution, and incubated for at least $30 \mathrm{~min}$ at $4{ }^{\circ} \mathrm{C}$ prior to flow cytometer analysis.

Parasites were sorted with a FACSAria II cell sorter (BD Bioscience) using BDFacsDiva software package (BD Biosciences). Gates were established based on the combination of FSC-W and FSC-H followed by SSC-W and SSC-H. Parasites were finally sorted based on the FITC intensity. Purity of cell population FITC low and FITC intermediate was above 97\%. After sorting, the parasites were analyzed by optical microscopy, electron microscopy, and Image Stream Analyzer.

\subsection{Flow cytometric immunofluorescence assay}

AMA live, TRYPO live and EPI fixed cultures prepared and adjusted to $1 \times 10^{7} / \mathrm{ml}$ earlier were stained with $200 \mu \mathrm{g} / \mathrm{ml}$ of FITC at $37{ }^{\circ} \mathrm{C}$ for $30 \mathrm{~min}$. Parasites were washed three times with $10 \%$ FBS-PBS $\left(900 \times g\right.$ for $10 \mathrm{~min}$ at $18{ }^{\circ} \mathrm{C}$ ) and resuspended in adjusted volume. AMA and TRYPO cultures were incubated at $37{ }^{\circ} \mathrm{C}$ for $60 \mathrm{~min}$, and EPI culture at $4{ }^{\circ} \mathrm{C}$ for $24 \mathrm{~h}$. After staining, the AMA and TRYPO cultures were run using the cytometer to check the proportion of amastigotes and trypomastigotes in every culture. Considering the proportions observed in the flow cytometer, cultures of AMA, TRYPO and EPI were mixed to obtain a single suspension with similar amounts of each form of parasite, approximately 33\%. The suspension was run into the cytometer until the desired fit was obtained.

Serum samples were inactivated at $56{ }^{\circ} \mathrm{C}$ for $30 \mathrm{~min}$ and kept at $-20{ }^{\circ} \mathrm{C}$ until use. The inactivated sera were diluted in $10 \%$ FBS-PBS and used to evaluate the presence of anti-T. cruzi antibodies by flow cytometry. All serum samples were filtered through a $0.22 \mu \mathrm{M}$ syringe filter to remove debris and platelets. The diluted and filtered serum was centrifuged at $13.000 \mathrm{rpm}$ for $10 \mathrm{~min}$ at room temperature.

The flow cytometry tests were performed as described by Cordeiro et al. (2001) and Vitelli-Avelar et al. (2007). Briefly, in a U bottom 96 well plate, $50 \mu$ of the single suspension containing live AMA, live TRYPO and fixed EPI (166,000 parasites/well) was incubated at $37{ }^{\circ} \mathrm{C}$ for $30 \mathrm{~min}$ in the presence of $50 \mu \mathrm{l}$ of individual serial serum dilutions (1/250 to $1 / 8000)$. After incubation with sera, parasites were washed once with $100 \mu \mathrm{l}$ of $10 \%$ FBS-PBS, and once with $200 \mu \mathrm{l}$ of $10 \%$ FBS-PBS ( $900 \times g$ for $10 \mathrm{~min}$ at $\left.18{ }^{\circ} \mathrm{C}\right)$, and the supernatant was removed. Parasites were re-incubated with $50 \mu \mathrm{l}$ biotin-conjugated anti-human IgG1 diluted in $10 \%$ FBS-PBS at $1 / 6400$, and $20 \mu \mathrm{l}$ of streptavidinphycoerytrin-SAPE diluted in $10 \%$ FBS-PBS at $1 / 400,37{ }^{\circ} \mathrm{C}$ for $30 \mathrm{~min}$. Parasites were washed twice as described above, fixed with $200 \mu \mathrm{l}$ of FACS fixing solution, and incubated for at least 30 min at $4{ }^{\circ} \mathrm{C}$ prior to their acquisition in the flow cytometer. Flow cytometric analyses were performed on the same day of the experiment.

\subsubsection{Flow cytometric data acquisition and analysis}

Flow cytometric measurements were performed on a FACSCalibur flow cytometer (Becton Dickinson, USA). The FlowJo software package was used for data acquisition, storage and analysis. Stained parasites were run in the cytometer, and 10,000 events per sample were acquired. Acquisition was performed by setting the size gain at E00 and granularity gain at 427, both on a log-scale. The fluorescence gains were set at 620 in log-scale mode for channel 1 (FITC) and at 500 in log-scale mode for channel 2 (avidin/PE).

To calculate the reactivity of the samples, parasites were identified on the basis of their specific forward (FSC) and side (SSC) light-scattering properties. Each evolutive form of T. cruzi was selected by gating on the fluorescence 1 (FITC) $\times$ FSC dot plot distribution. The relative avidin/PE fluorescence intensity for each evolutive form was analyzed using a single histogram representation. A control marker of up $2 \%$ of the parasites that were fluorescence positive (PPFP) was set up on the internal control histogram for nonspecific binding of avidin/PEconjugated anti-human IgG1. This marker was used to determine for each serum sample the percentage of positive fluorescent parasites (PPFP).

\subsection{Statistical analysis}

The performance of FC-ATE was assessed by the following indexes: sensitivity ([true positives / true positives + false negatives] $\times 100$ ) and specificity ([true negatives / true negatives + false positives $] \times 100)$. The data were analyzed by receiver operating characteristic (ROC) curve analysis, using the "Med Calc Statistical" program. The area under the curve provided the global test accuracy, which was classified as: low (0.51-0.61), moderate (0.62-0.81), elevated (0.820.99 ) and outstanding (1.0) (Swets, 1988). ROC curve also indicated a cut-off point related to less erroneous results. Comparative analysis of average reactivity of IgG1 antiT. cruzi antibodies between the $\mathrm{NCH}_{1}$ and $\mathrm{CH}_{1}$ groups was performed by the non-parametric test of Mann-Whitney using the "Graph Pad Prism 5" software.

\section{Results}

\subsection{FC-ATE can accurately discriminate the three evolutive forms of T. cruzi stained with FITC}

AMA, TRYPO and EPI preparations showed similar forward (FSC) and side (SSC) light-scattering properties in dot plot distribution (data not shown). Thus, in order to discriminate the parasite forms the use of differential staining with FITC was proposed. The separation of the three evolutive forms was obtained only under different temperatures and times of incubation, TRYPO + AMA at $37{ }^{\circ} \mathrm{C}$ for 60 min and EPI at $4{ }^{\circ} \mathrm{C}$ for $24 \mathrm{~h}$. After incubation, parasites were run and analyzed in the FSC $\times$ FITC dot plot. Histograms are shown in Fig. 1A. AMA + TRYPO mixture preparation exhibited two distinguished populations, based on the intensity of fluorescence, low (LOW) intensity and intermediate (INT) intensity. EPI preparation showed a homogenous high intensity population (HIGH).

Fig. 1. FC-ATE can accurately discriminate the three evolutive forms of T. cruzi stained with FITC. (A) Dot plot analysis of representative culture AMA + TRYPO and culture of EPI distribution in size (FSC) versus granularity (SSC) and fluorescence (FITC) versus size (FSC) after stained with FITC. Representative histograms of cultures of AMA + TRYPO and EPI after stained with FITC. The three evolutive forms were stained with $200 \mu \mathrm{g} / \mathrm{ml} \mathrm{FITC}$ and AMA + TRYPO was incubated at $37{ }^{\circ} \mathrm{C}$ for 60 min and EPI was incubated at $4{ }^{\circ} \mathrm{C}$ for $24 \mathrm{~h}$. (B) Detailed morphometric cellular analysis of the morphology (M), granularity ( $\mathrm{G}$ ), nucleous ( $\mathrm{N}$ ) and merges $\mathrm{N}+\mathrm{G}$ and $\mathrm{M}+\mathrm{N}+\mathrm{G}$ obtained by ImageStream Flow cytometry and photographs by optical microscopy $(100 \times$ objectives $)$ and electron microscopy $(12,000 \times$ objectives/bar $=1 \mu \mathrm{m}$ and $30,000 \times$ objectives/bar $=500 \mathrm{~nm})$ the AMA and TRYPO after the sorting of parasites. 
A
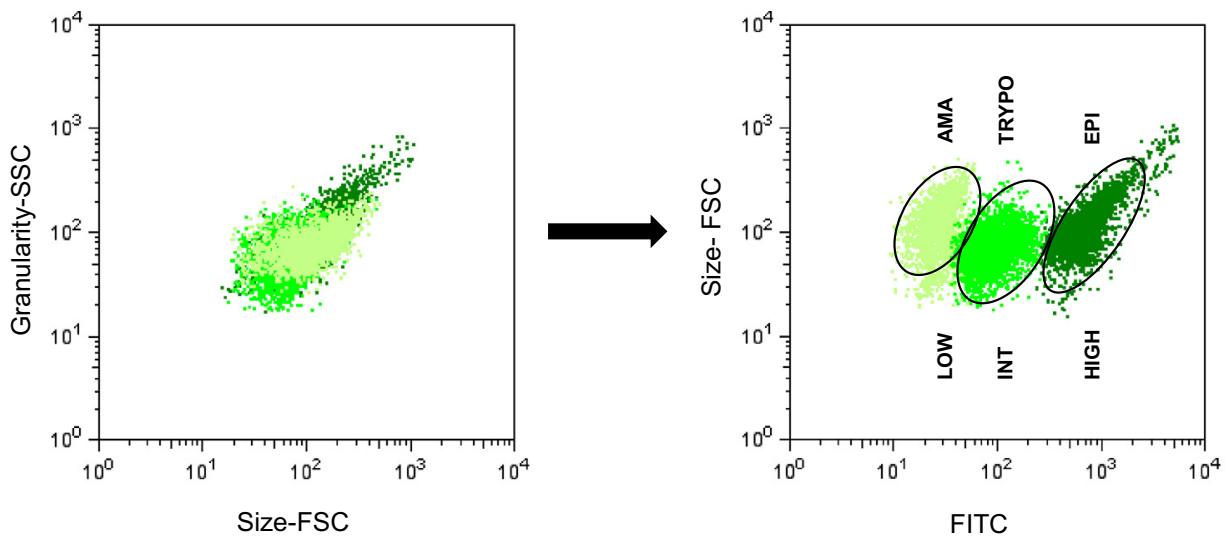

B

Internal Control

Positive Control

Negative Control
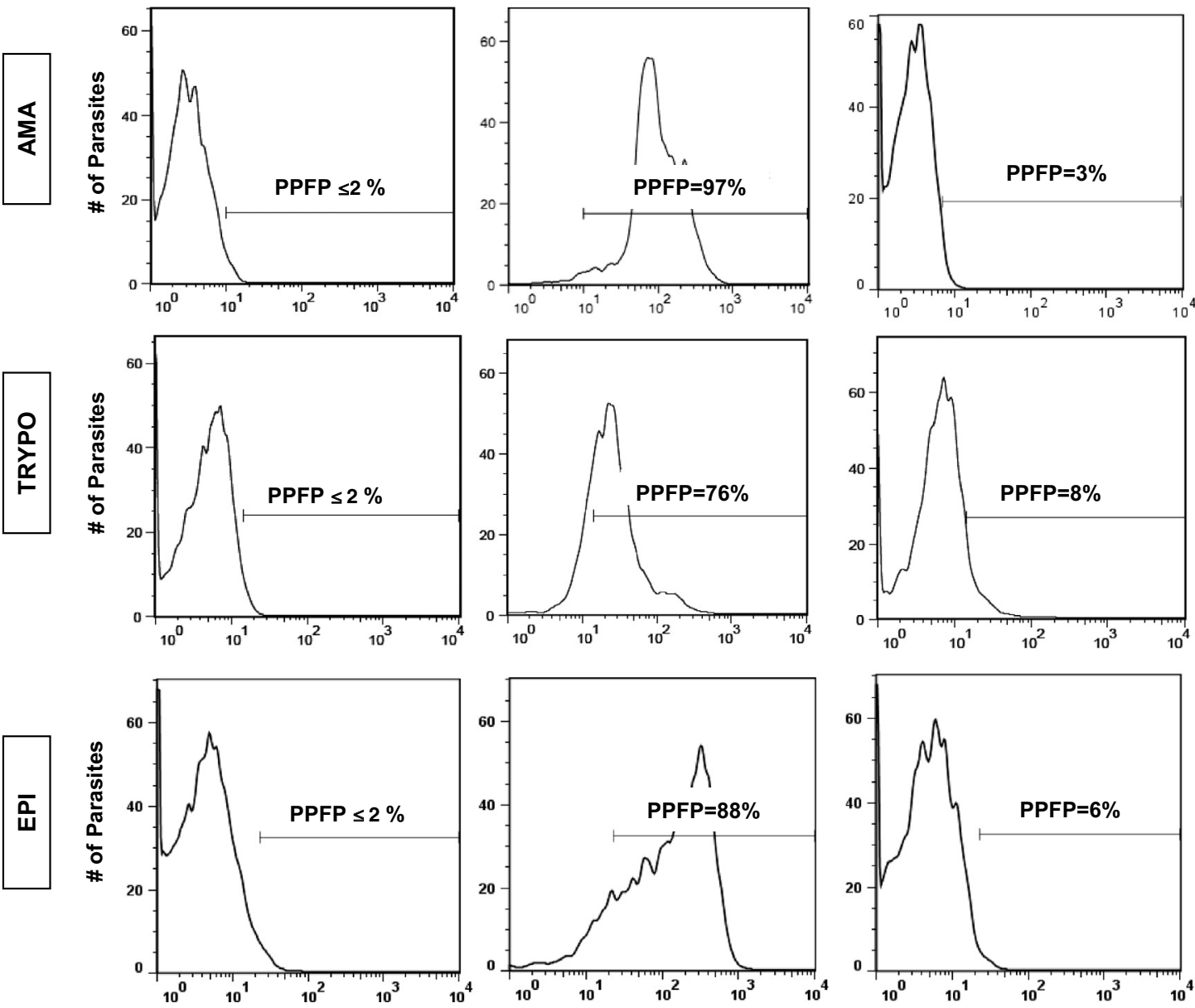

Avidin/PE

Fig. 2. Determination of the IgG1 anti-live AMA, anti-live TRYPO, anti-fixed EPI reactivities. (A) Dot plot analysis of representative AMA, TRYPO and EPI distribution based on size (FSC) versus granularity (SSC) and fluorescence (FITC) versus size (FSC) after mixing the three stained evolutive forms. AMA showed low fluorescence (LOW), TRYPO intermediated fluorescence (INT) and EPI high fluorescence (HIGH) in graphs FITC $\times$ FSC. (B) Single histograms represent PPFP values for avidin-PE conjugated anti-human IgG1 internal control, positive control and negative control. A control marker of up $2 \%$ of the parasites that were fluorescence positive was set up on the internal control histogram. This marker was used to determine PPFP for each sample. 
To better characterize the double population seen in AMA + TRYPO preparation, a cell sorting in FACSAria cytometer in order to isolate both populations was performed. After sorting, the separated populations were further analyzed by optical microscopy, electronic microscopy, and ImageStream Analyzer (Fig. 1B). Thus, it was identified that the INT population refers to TRYPO forms and that the LOW population refers to the AMA forms.

\subsection{Determination of the IgG1 anti-live AMA, anti-live TRYPO, and anti-fixed EPI reactivities}

The profile of the parasite preparation of equivalent quantities of the three stained evolutive forms showed the profiles displayed in Fig. 2A. In the FITC $\times$ FSCgraph, an evident differential staining of AMA, TRYPO and EPI was seen. Due to this clear distinction, was possible simultaneously investigate antibodies specific to each evolutive forms of $T$. cruzi. After selecting the specific population of parasite form in the FITC $\times$ FSC dot plot distribution (Fig. 2A), reactivity of positive and negative controls with AMA, TRYPO and EPI was verified (Fig. 2B). Using a control set up marker of $2 \%$ of PPFP in the internal control histogram, the PPFP of each individual sample was established. Positive control values of PPFP were 97\%, 76\% and $88 \%$ in AMA, TRYPO and EPI, respectively, while in negative controls, PPFP were $3 \%, 8 \%$ and $6 \%$, respectively. Interestingly, the results also showed differences in the reactivity of positive sera when we compared the evolutive forms, presenting higher percentage with AMA (97\%) than with EPI (88\%), and lowest percentage with TRYPO (76\%).

\subsection{Performance of FC-ATE in the discrimination of serum samples from the $\mathrm{NCH}_{1}$ and $\mathrm{CH}_{1}$ patients}

The results of the standardization and performance analysis of FC-ATE reactivity of the serum samples of Group 1 with AMA, TRYPO and EPI are showed in Fig. 3. The analysis of the mean values of PPFP in the titration curves showed significant statistical difference between the reactivity of serum samples from the $\mathrm{CH}_{1}$ and $\mathrm{NCH}_{1}$ patients in all dilutions (1/250 to 1/ 8000 ) tested, and with the three evolutive forms of T. cruzi (Fig. 3A upper). To select the ideal dilution to discriminate $\mathrm{CH}_{1}$ from $\mathrm{NCH}_{1}$, the higher mean percentage variation ( $\triangle \mathrm{PPFP}$ ) between $\mathrm{CH}_{1}$ from $\mathrm{NCH}_{1}$ was determined (Fig. 3A down). The results showed that three dilutions $(1 / 250,1 / 500$ and $1 / 1000)$ out of the six dilutions tested $(1 / 250$ to $1 / 8000)$ better segregated the groups of $\mathrm{CH}_{1}$ and $\mathrm{NCH}_{1}$, as demonstrated in the dotted rectangle. However, the dilution 1/1000 with AMA, 1/250 with TRYPO and EPI presented the best performance in the discrimination of the two groups.

Using ROC curves built with the values of PPFP from individual samples of $\mathrm{CH}_{1}$ and $\mathrm{NCH}_{1}$, in the three selected dilutions (1/250, 1/500 and 1/1000), FC-ATE results showed excellent accuracy in all three dilutions, since the values of area under the curve were 1.0 or very close to 1.0 (data not shown). The ROC curve also showed the values of sensitivity and specificity, and their respective cut-offs for each of the three dilutions tested with AMA, TRYPO and EPI (Fig. 3B). This analysis demonstrated that the dilution 1/1000 with a cut-off of $40 \%$ for AMA and the dilution 1/250 with a cut-off of $20 \%$ for TRYPO and
EPI presented $100 \%$ of sensitivity and specificity, in addition to a lower profile of dispersion among reactivity values in each group.

These data defined the criteria for the use of the FC-ATE technique in the diagnosis and post-therapeutic monitoring of Chagas disease.

\subsection{Applicability of FC-ATE in diagnosis of Chagas disease}

The applicability of FC-ATE for the diagnosis of Chagas disease was evaluated in serum samples of Group 2 in reaction with AMA, TRYPO and EPI (Fig. 4). This analysis was done considering the better serum dilutions and the cut-off previously established. Data demonstrate that $94 \%$ of the serum samples from the $\mathrm{NCH}_{2}$ patients were non-reactive with AMA and TRYPO, and $88 \%$ were non-reactive with EPI. All the serum samples of the $\mathrm{CH}_{2}$ patients were reactive with AMA, TRYPO and EPI (Fig. 4A).

Serum samples that were positive in the $\mathrm{NCH}_{2}$ group were from patients with VL and ACL. In the VL group, 90\% were nonreactive to AMA and TRYPO, and $80 \%$ were non-reactive to EPI. In the ACL group, $80 \%$ were non-reactive to AMA and TRYPO, and $60 \%$ were non-reactive to EPI (Fig. 4B). A good applicability of FC-ATE in the diagnosis of Chagas disease was demonstrated, mainly with AMA and TRYPO forms of T. cruzi.

\subsection{Applicability of FC-ATE in the post-therapeutic monitoring of Chagas disease}

The applicability of the FC-ATE technique in post-therapeutic monitoring of Chagas disease was evaluated in serum samples of Group 3 in reaction with AMA, TRYPO and EPI (Fig. 5). Fig. 5A showed that serum samples of the NT and TNC groups were reactive with the three evolutive forms of T. cruzi. In TC, $100 \%$ were non-reactive to AMA, 93\% to TRYPO, and 96\% to EPI (Fig. 5A). Overall, the outstanding applicability of the FC-ATE technique was demonstrated, now in post-therapeutic monitoring of Chagas disease, mainly with AMA forms, since they were able to discriminate completely the NT and TNC patients from the TC patients.

Some serum samples of the NT $(n=12)$, TNC $(n=10)$, and TC $(n=21)$ groups have been tested by the methodologies of FC-ALTA and FC-AFEA in comparison with FC-ATE. We considered the dilutions and cut-off standardized for each methodology: FC-ALTA, dilution of $1 / 256$ and cut-off of 20\%; FC-AFEA, dilution of $1 / 2048$ and cut-off of $60 \%$, and FC-ATE, dilution of $1 /$ 250 and cut-off of $20 \%$ for TRYPO and EPI. Data showed that although these techniques exhibit different reaction magnitudes, they presented $100 \%$ of categorical correlation (Fig. 5B).

\section{Discussion}

Different techniques used for both, diagnosis and posttherapeutic monitoring of Chagas disease have several methodological limitations and lack of sensitivity and specificity. The chronic phase of the Chagas disease is characterized by low parasitemia and constant presence of antibodies, reasons why the serological methods are the main choice for diagnosis during this phase of the infection (Portela-Lindoso and Shikanai-Yasuda, 2003). These methods present high sensitivity and specificity, fast, cheap and easy to be used. However serological tests may frequently display cross-reactivity against other pathogens such as those belonging to the Trypanosomatidae family, especially 
A
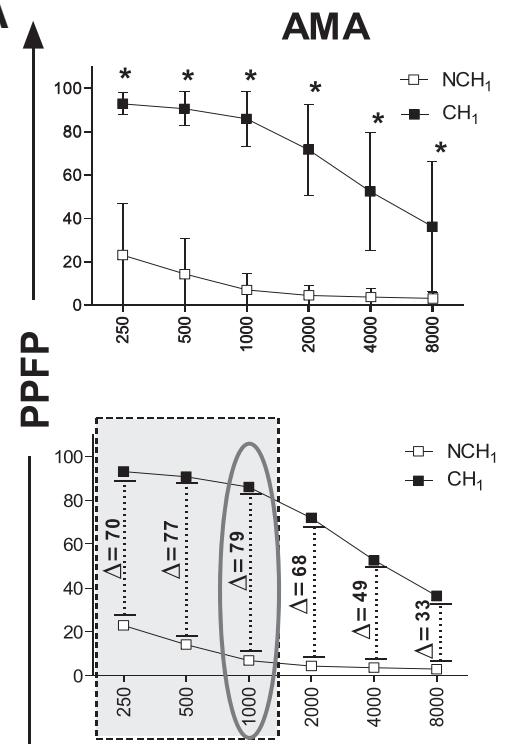

TRYPO

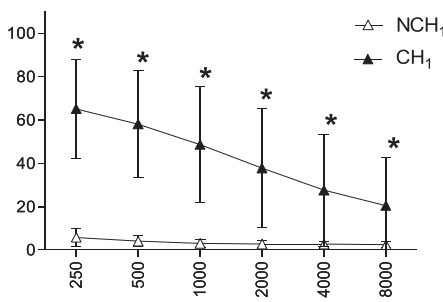

EPI

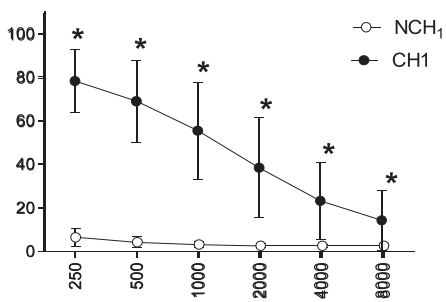

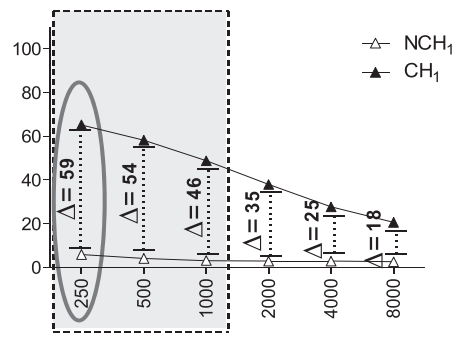

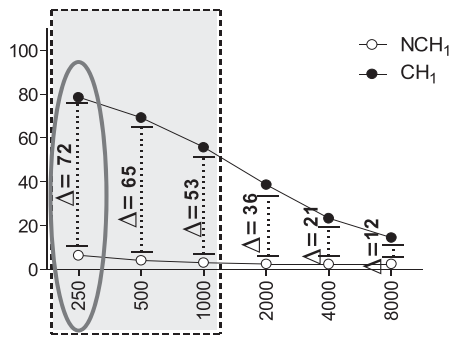

\section{Serum dilution}

B
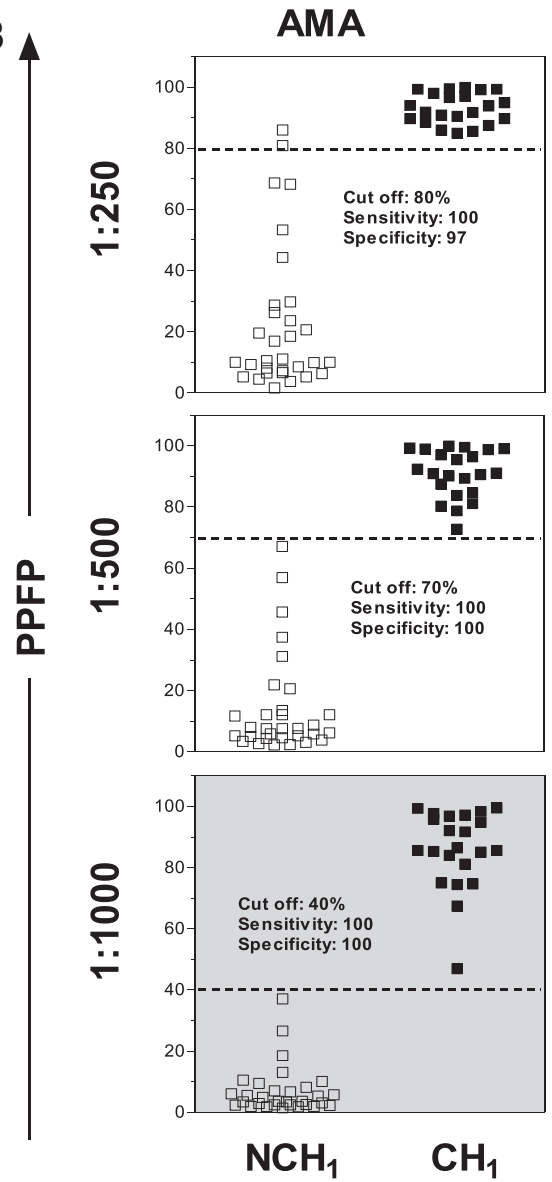

TRYPO
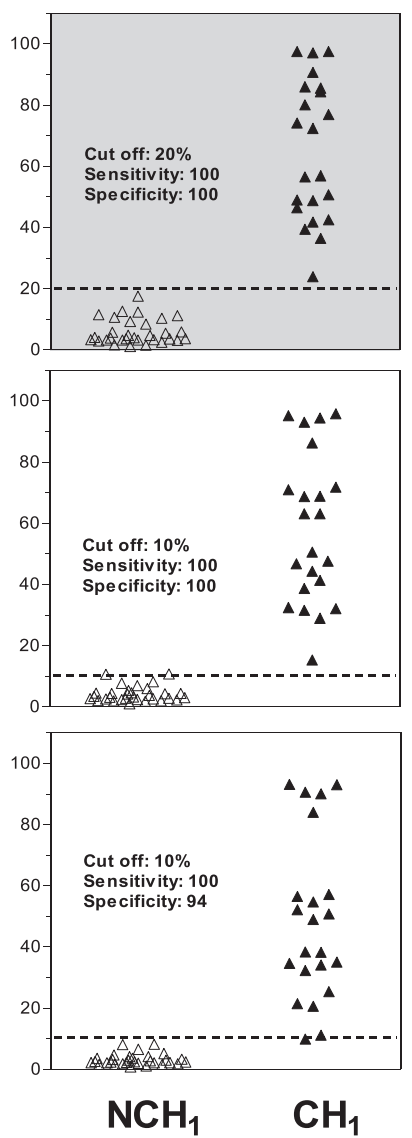

EPI
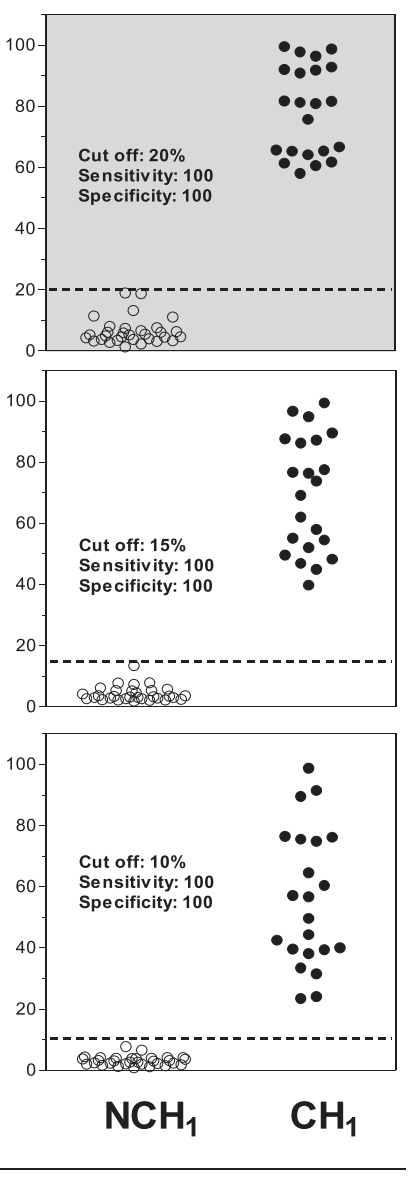

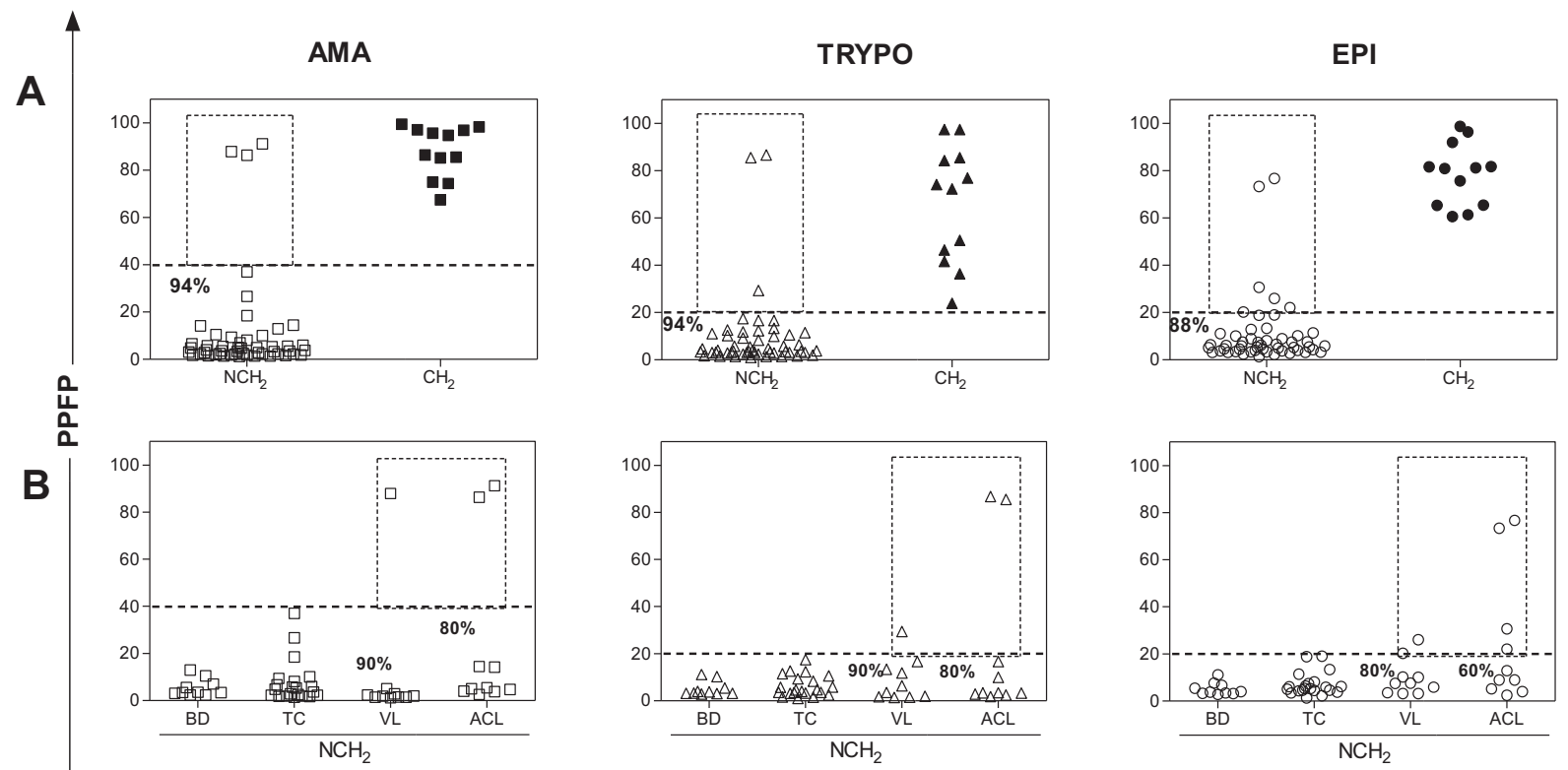

Fig. 4. Applicability of the FC-ATE in diagnosis of Chagas disease. (A) FC-ATE of individual serum samples from the $\mathrm{NCH}_{2}$ (white symbol) and $\mathrm{CH}_{2}$ (black symbol) patients at 1/1000 dilution for AMA (square symbol) and 1/250 dilution for TRYPO (triangle symbol) and EPI (circle symbol). (B) FC-ATE of individual serum samples from $\mathrm{NCH}_{2}$, including the BD, TC, VL and ACL (all groups white symbol) patients at 1/1,000 dilution for AMA (square) and 1/250 dilution for TRYPO (triangle) and EPI (circle). The results were expressed as PPFP. The dotted line represented the cut-off between negative and positive values with each evolutive form of T. cruzi: AMA cutoff of $40 \%$, TRYPO cut-off of $20 \%$ and EPI cut-off of $20 \%$. The dotted rectangles select the false positive results.

with Leishmania spp. (Vexenat et al., 1996). Another drawback is related to its use in post-therapeutic monitoring of disease due to the long term required for host seroreversion after the etiologic treatment of T. cruzi infection (Viotti et al., 1994; Cançado, 2002; Coura and Castro, 2002; Lana and Martins-Filho, 2009; Machado-de-Assis et al., 2012).

Flow cytometry is a non-conventional serological method used for detection of anti-live trypomastigote antibodies (FCALTA) and anti-fixed epimastigote antibodies (FC-AFEA) that have been described as new approaches for diagnosis and posttherapeutic monitoring of Chagas disease. However, as FCALTA presents operational difficulties, recently, a flow cytometric assay to assess the $\operatorname{IgG}$ reactivity to fixed $T$. cruzi epimastigotes (FC-AFEA) was standardized. This additional methodology, although had displayed a good performance in the diagnostic and pos-therapeutic monitoring of Chagas disease, still presents limitations regarding sensitivity and specificity (Vitelli-Avelar et al., 2007; Matos et al., 2011). These limitations of FC-ALTA and FC-AFEA are related to multifactorial reasons such as type, source and purity of the antigen employed as well as the detection system intrinsic to each technology.
In this context, this current work searched for ideal antigenic preparation for optimum or at least better performance of the serological tests for the diagnosis and pos-therapeutic monitoring of Chagas disease, through evaluation of anti-live amastigote, anti-live trypomastigote, and anti-fixed epimastigote IgG1 antibodies by flow cytometry. Primarily, we looked for a distinctive profile of the three evolutive forms of $T$. cruzi after reading on the flow cytometer to assess the reactivity of the serum samples with each form individually. Therefore, differential fluorescence stained with FITC to the three evolutive forms of T. cruzi was standardized. Interestingly, in the mixture of AMA + TRYPO culture, it was identified, through sorting, microscopy, and flow cytometry imaging that AMA was the evolutive form that presented lower fluorescence, while TRYPO presented higher fluorescence. The differential staining of these two forms of the parasite may be due to the higher density of proteins and larger amount of membrane presented in TRYPO compared with AMA. Next, the reactivity of the positive and negative control serum samples with AMA, TRYPO and EPI individually was evaluated. It was verified that the reactivity between the controls and the three evolutive forms of $T$. cruzi were distinct, what there was been previously observed by Cordeiro et al. (2001).

Fig. 3. Performance of FC-ATE in the discrimination of serum samples of patients $\mathrm{NCH}_{1}$ and $\mathrm{CH}_{1}$. (A) Titration curves $(1 / 250$ to $1 / 8000)$ showing the average percentage of IgG1 anti-live AMA (square symbol), anti-live TRYPO (triangle symbol), anti-fixed EPI (circle symbol) reactivities of sera from $\mathrm{NCH}_{1}\left(\right.$ white symbol) and $\mathrm{CH}_{1}$ (black symbol). The results of IgG1 were expressed in PPFP. Upper showed standard deviation value of PPFP and statistical difference in all tested dilutions, $\mathrm{p} \leq 0,05(*)$; down showed the differences on the reactivity percentage between groups $(\Delta)$, inserted in the curve. The gray rectangles selected the three serum dilutions that showed the highest segregation of reactivity between groups. The dotted circles selected the best dilutions that showed segregation of reactivity between $\mathrm{NCH}_{1}$ and $\mathrm{CH}_{1}$. (B) Analysis of IgG1 reactivity of individual samples of the $\mathrm{NCH}_{1}$ patients (white symbol) and $\mathrm{CH}_{1}$ (black symbol) in the dilutions $(1 / 250 ; 1 / 500$ and $1 / 1000)$ with $\mathrm{AMA}$ (square symbol), TRYPO (triangle symbol) and EPI (circle symbol). The cut-offs, sensitivity and specificity were selected in the ROC curve analysis. The gray charts selected the best dilutions and cut-offs that showed better segregation of the reactivity between groups to be applied in the discrimination of serum samples of the $\mathrm{NCH}_{1}$ and $\mathrm{CH}_{1}$ patients. 

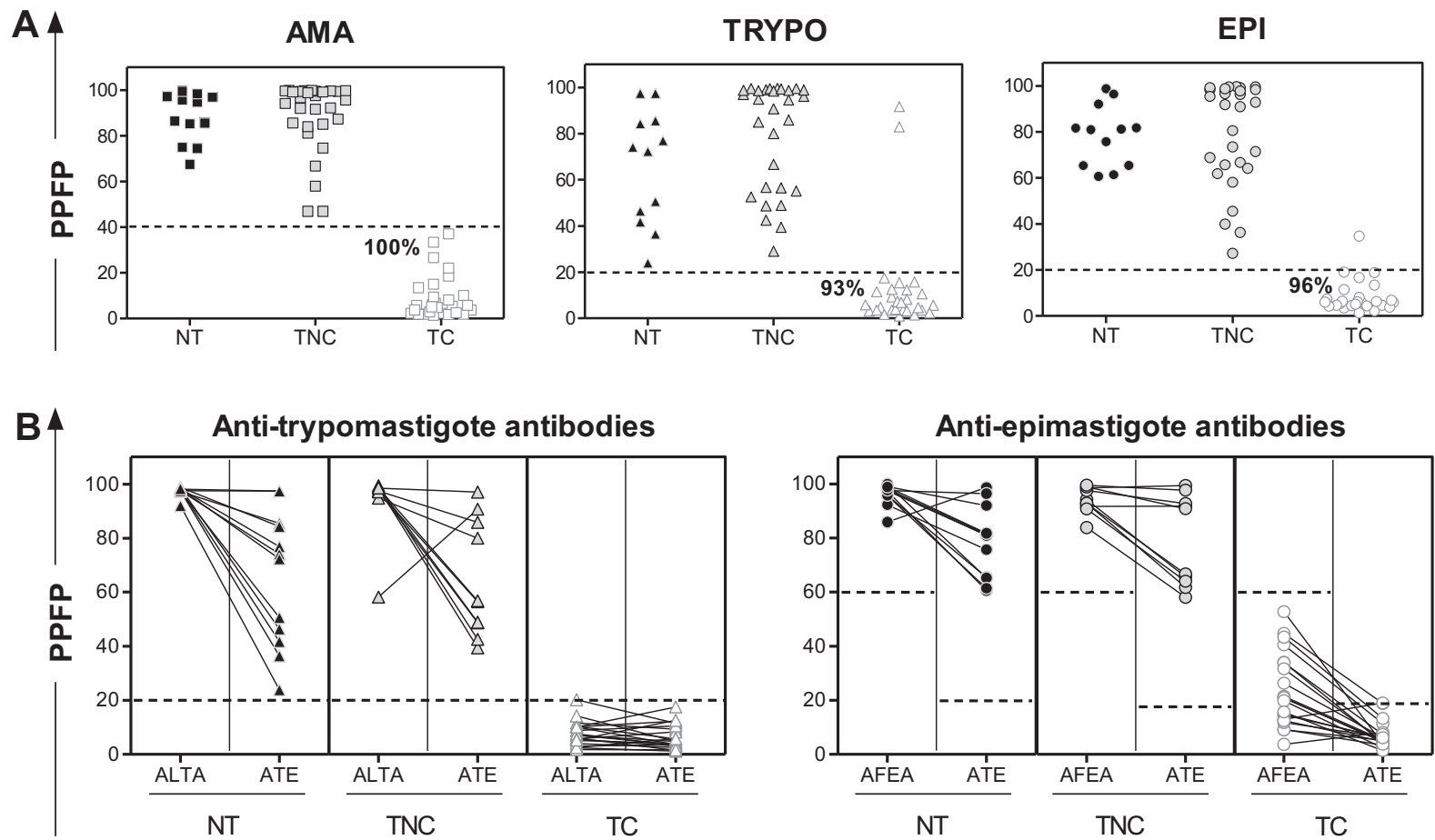

Fig. 5. Applicability of FC-ATE in the post-therapeutic monitoring of Chagas disease. (A) FC-ATE of individual serum samples from the NT (black symbol), TNC (gray symbol) and TC (white symbol) individuals at 1/1000 dilution for AMA (square symbol) and 1/250 dilution for TRYPO (triangle symbol) and EPI (circle symbol). The results were expressed as PPFP. The dotted line represented the cut-off between negative and positive values with each evolutive form of $T$. cruzi: AMA cut-off of $40 \%$, TRYPO cut-off of $20 \%$ and EPI cut-off of $20 \%$. (B) Scatter graphs showed reactivity in PPFP in methodologies FC-ALTA (triangle symbol), FC-AFEA (circle symbol) and FCATE (triangle symbol for TRYPO and circle symbol for EPI) for the groups NT (black symbol), TNC (gray symbol) and TC (white symbol) at $1 / 256$ dilution for FC-ALTA, $1 /$ 250 dilution for FC-ATE and 1/2048 dilution for FC-AFEA. The reactivity FC-ALTA $\times$ FC-ATE and reactivity FC-AFEA $\times$ FC-ATE, for comparative analysis, were indicated by connecting lines. The dotted line represents the cut-off between negative and positive values of the serum samples: TRYPO (FC-ALTA) cut-off of 20\%, TRYPO (FC-ATE) cut-off of $20 \%$, EPI (FC-AFEA) cut-off of $60 \%$ and EPI (FC-ATE) cut-off of $20 \%$.

In the next step it was purposed the analysis of the performance of FC-ATE for the diagnosis of Chagas disease. The indexes of reactivity demonstrated the outstanding performance of FC-ATE in the discrimination of $\mathrm{NCH}_{1}$ and $\mathrm{CH}_{1}$ sera, with the area under curve equal to 1 , and values of sensitivity and specificity of $100 \%$. In addition, the dilutions and cut-offs for each evolutive form of T. cruzi used in FC-ATE were established. The results demonstrated that FC-ATE showed higher sensitivity than parasitological methods such as hemoculture (0 to 55\%) (Chiari et al., 1989; Brener et al., 1993), xenodiagnosis (13 to 50\%) (Cerisola et al., 1974; Pereira et al., 1996; Schenone et al., 2000), PCR (45-86\%) (Gomes et al., 1998; Britto, 2009), and higher or similar sensitivity than conventional serological methods (95 to 100\%) (Oelemann et al., 1998). Furthermore, the sensitivity values found were higher than those presented by FC-AFEA, 82\% (Vitelli-Avelar et al., 2007), and FCAFEA with modifications (sensitivity of $97.1 \%$, and specificity of 95.4\%) (Matos et al., 2011). Interestingly, in FC-ATE, AMA was more reactive (serum dilution 1:1000), while the better reactivity with TRYPO and EPI was observed in the serum dilution of 1:250. In disagreement with these results, some previous studies evaluating FC-ALTA and FC-AFEA demonstrated that EPI was always more reactive than TRYPO. In FC-ALTA, the dilution and cut-off established were 1:256 and 20\%, respectively (Martins-Filho et al., 1995, 2002; Cordeiro et al., 2001), in FCAFEA 1:2048, 1:4096 and 60\%, respectively (Vitelli-Avelar et al., 2007; Matos et al., 2011). These data suggest the existence of competition among antigens and antibodies with higher affinity for AMA. AMA forms have been used as antigenic sources in serological tests for Chagas disease, and also showed higher reactivity than antigens of EPI (Cerisola et al., 1971; Primavera et al., 1990; Matsumoto et al., 1993).

After the analysis of the performance of FC-ATE, the applicability of this new assay in the diagnosis of Chagas disease was evaluated. According to the standard criteria for this technique, and considering the comparison between the $\mathrm{NCH}_{2}$ and $\mathrm{CH}_{2}$ groups, it was verified that $100 \%$ of the $\mathrm{CH}_{2}$ serum samples were positive for all three evolutive forms of T. cruzi, $94 \%$ of the serum samples of the $\mathrm{NCH}_{2}$ individuals were negative with AMA (PPFP $<40 \%$ ) or TRYPO (PPFP $<20 \%$ ), and $88 \%$ of the $\mathrm{NCH}_{2}$ individuals were negative with EPI (PPFP $<20 \%$ ), what confirmed the good performance of FC-ATE in the diagnosis of Chagas disease.

Other studies using FC-ALTA and FC-AFEA also demonstrated the good applicability of flow cytometry in the diagnosis of Chagas disease (Martins-Filho et al., 1995; Cordeiro et al., 2001; Vitelli-Avelar et al., 2007; Matos et al., 2011). Cordeiro et al. (2001) using a fixed EPI, fixed TRYPO and live TRYPO as antigens demonstrated that independent of the parasite preparation used, the mean values of PPFP were higher for chagasic pooled sera in comparison to non-chagasic, which is in disagreement with the results obtained by FC-ATE where the majority of the sera from the $\mathrm{NCH}$ patients showed negative values of reactivity against the three evolutive forms of T. cruzi. Studies using FC-AFEA for the diagnosis of Chagas disease 
showed that $100 \%$ of samples of the $\mathrm{NCH}$ patients were negative, and $82 \%$ of samples of the $\mathrm{CH}$ group were positive (Vitelli-Avelar et al., 2007). On the other hand, in the modified FC-AFEA assay, 95.4\% of the serum samples from non-infected group, and TC were negative, and $95.6 \%$ of the serum samples from the chagasic and TNC groups were positive (Matos et al., 2011). These data were similar to those found in FC-ATE, but the results in relation to reactivity of the serum samples from $\mathrm{CH}$ were higher than for FC-AFEA, since $100 \%$ of the samples were positive, regardless of the antigen preparation used.

One of the advantages to use flow cytometry for the diagnosis of Chagas disease is its ability to detect 10.000 fluorescent parasites and all the parameters can be measured, stored and simultaneously analyzed in computer programs flow cytometer.

In this study, serum samples of the $\mathrm{NCH}_{2}$ patients are from a pooled population that includes individuals with other relevant infectious/parasitic diseases (patients with VL and ACL), as well as $\mathrm{BD}$ and TC. Note that the $\mathrm{NCH}_{2}$ samples that showed falsepositive results in FC-ATE are from patients with VL and ACL. The false-positive results found may be due to shared antigens among the Trypanosomatideos, triggering frequently a cross reactivity between $T$. cruzi and Leishmania spp. in serological assays, a phenomenon previously described (Vexenat et al., 1996; Gadelha et al., 2003). Despite the high sensitivity of different serological tests for the diagnosis of Chagas disease, the cross-reactivity mainly with sera from the VL and ACL patients is still frequent (Nakazawa et al., 2001; Amato Neto et al., 2005; Berrizbeitia et al., 2006, 2012). Berrizbeitia et al. (2012) demonstrated that despite the high sensitivity of this test for the diagnosis of Chagas disease, employing secreted/ excreted protein of epimastigotes, its specificity was only $88 \%$, and cross-reactions were observed with Ascaris spp. and Leishmania spp. The cross-reactivity with sera from patients VL and ACL was also observed in TESA-blot, using secreted/ excreted antigens of trypomastigotes (Nakazawa et al., 2001; Amato Neto et al., 2005; Berrizbeitia et al., 2006). Nevertheless, studies using secreted/excreted antigens, recombinant antigens or synthetic peptides have lower percentage of crossreactivity with sera from patients with other infectious/ parasitic diseases (Gomes et al., 2001; Umezawa et al., 2001; Caballero et al., 2007; Flores-Chávez et al., 2010).

In our study, FC-ATE showed false-positive results comparable or lower than FC-AFEA (Vitelli-Avelar et al., 2007; Matos et al., 2011). In FC-AFEA, 52\% of the samples from patients with VL were false-positives (Vitelli-Avelar et al., 2007). When this technique was modified (Matos et al., 2011), the percentage of the false-positive results was $54 \%$ in patients with VL, and $21 \%$ in patients with ACL. In the current assay, $10 \%$ of patients with VL, and 20\% with ACL were false-positives in the AMA and TRYPO tests, while with EPI these percentages were $20 \%$ for the VL and $40 \%$ for the ACL patients.

A relevant data of this study was that AMA and TRYPO showed a lower percentage of false-positive results in comparison with EPI. This fact can be possible since AMA and TRYPO are present in the tissues and blood of the vertebrate host and thus, these forms are more specific, whereas EPI is present only in the invertebrate host, then, cross-reactivity may occur more frequently with other pathogens (Primavera et al., 1990). However, Berrizbeitia et al. (2004) observed percentages of specificity similar to fixed AMA, fixed TRYPO, and fixed EPI. Another fact that may explain the occurrence of false-positive with AMA and TRYPO in FC-ATE is that some leishmaniasis patients ( $\mathrm{VL}=01$ and $\mathrm{ACL}=02$ ) may have co-infection with $T$. cruzi, because in our current assay they were positive for the three evolutive forms of the parasite.

Considering the promising results with FC-ATE for diagnosis, its applicability was also essayed for the post-therapeutic monitoring of Chagas disease, since one of the major challenges regarding the evaluation of treatment effectiveness in the chronic phase of infection is the lack of a successful laboratorial method for use as cure criteria. Thus, there is an urgent need for the development of serological tests to be employed in the posttherapeutic monitoring of Chagas disease. In this context, it was recently showed that the determination of anti-T. cruzi antibodies by non-conventional flow cytometry methods is a promising tool to monitor treatment efficacy in Chagas disease patients (Martins-Filho et al., 1995, 2002; Vitelli-Avelar et al., 2007). The performance analysis of FC-ATE demonstrated that $100 \%$ of the serum samples of the patients NT and TNC were positive for the three evolutive forms of T. cruzi, $100 \%$ of the samples of the TC were negative with AMA, 93\% of the TC were negative with TRYPO, and 96\% with EPI. These findings demonstrated an outstanding performance of FC-ATE for the post-therapeutic monitoring of Chagas disease. The results were consistent with those found for FC-ALTA and FC-AFEA (Martins-Filho et al., 1995, 2002; Vitelli-Avelar et al., 2007; Matos et al., 2011). Considering that the results found in FC-ATE were consistent with those detected for FC-ALTA and FC-AFEA, a comparative analysis of these both techniques with FC-ATE was performed using serum samples from the NT, TNC and TC groups. When FC-ALTA and FCATE or FC-AFEA and FC-ATE performances were compared, we observed that different magnitudes of reactivities among groups were different. Most samples tested in FC-ATE reacted similar to or less than the other two methods. We believe that the contradictory results among the available tests were not surprising because the antigenic preparations were different, and consequently, have different affinities for both, specific and nonspecific antibodies. Nevertheless, these techniques presented categorical correlation, which means that all serum samples of the NT and TNC patients were positive and that all serum samples of the TC patients were negative, which demonstrated again the applicability of FC-ATE in post-therapeutic monitoring of Chagas disease.

An important finding of this study is that AMA form is able to produce a complete discrimination between the NT and TNC patients from the TC patients. This finding reveals that AMA may be a promising antigen to the development of new serological tests for the diagnosis and post-therapeutic monitoring of Chagas disease.

In conclusion, this study developed a new method using a broader antigen preparation to detect simultaneously the reactivity against the three evolutive forms of $T$. cruzi employing a differential fluorescent staining of the parasites with a promising performance for the diagnosis and post-therapeutic monitoring of Chagas disease.

\section{Acknowledgments}

This work was supported by UFOP, CPqRR/FIOCRUZ, Fundação de Amparo à Pesquisa de Minas Gerais (FAPEMIG), Conselho 
Nacional de Desenvolvimento Científico e Tecnológico (CNPq), Coordenação de Aperfeiçoamento de Pessoal de Nivel Superior (CAPES). We also thank the program for technological development in tools for health PDTISFIOCRUZ for use of its facilities. We gratefully acknowledge to Dr. Fernanda Freire Campos Nunes and Dr. Márcio Sobreira Silva Araújo for providing us with technical support during the experiments, Dr. Célia Maria Ferreira Gontijo for providing us with serum samples, and Dr. Silvana Maria Elói Santos for providing us with serum samples and for performing a critical review of the manuscript.

\section{References}

Amato Neto, V.,De Marchi, C.R.,Ferreira, C.S.,Ferreira, A.W., 2005. Observations on the use of TESA blot for the serological diagnosis of Chagas' disease. Rev. Soc. Bras. Med. Trop. 38, 534.

Araujo, F.G., Guptill, D., 1984. Use of antigen preparations of the amastigote stage of Trypanosoma cruzi in the serology of Chagas' disease. Am. J. Trop. Med. Hyg. 33, 362.

Arce-Fonseca, M., Ramos-Ligonio, A., López-Monteón, A., Salgado-Jiménez, B. Talamás-Rohana, P.,Rosales-Encina, J.L., 2011. A DNA vaccine encoding for TcSSP4 induces protection against acute and chronic infection in experimental Chagas disease. Int. J. Biol. Sci. 7, 1230.

Berrizbeitia, M.,Ndao, M.,Gottschalk, M.,Aché, A.,Vásquez, F.,Lacouture, S.,Medina, M.,Ward, B., 2004. Development and comparison of enzyme immunoassays for diagnosis of Chagas' disease using fixed forms of Trypanosoma cruzi (epimastigotes, amastigotes, and trypomastigotes) and assessment of antigen stability for the three assays. J. Clin. Microbiol. 42, 1766.

Berrizbeitia, M.,Ndao, M.,Bubis, J.,Gottschalk, M.,Aché, A.,Lacouture, S., Medina, M.,Ward, B.J., 2006. Purified excreted-secreted antigens from Trypanosoma cruzi trypomastigotes as tools for diagnosis of Chagas' disease. J. Clin. Microbiol. 44, 291.

Berrizbeitia, M., Figueroa, M., Ward, B.J., Rodríguez, J.,Jorquera, A., Figuera, M.A., Romero, L.,Ndao, M., 2012. Development and application of an ELISA assay using excretion/secretion proteins from epimastigote forms of T. cruzi (ESEA Antigens) for the diagnosis of Chagas disease. J. Trop. Med. 1.

Bertelli, M.S., Golgher, R.R., Brener, Z., 1977. Intraspecific variation in Trypanosoma cruzi: effect of temperature on the intracellular differentiation in tissue culture. J. Parasitol. 63, 434.

Brener, Z., 1965. Comparative studies of different strains of Trypanosoma cruzi. Ann. Trop. Med. Parasitol. 59, 19

Brener, Z., 1973. Biology of Trypanosoma cruzi. Annu. Rev. Microbiol. 27, 347.

Brener, Z., Cançado, J.R.,Galvão, L.M.,Luz, Z.P.,Filardi, L.S.,Pereira, M.E.,Santos, L.M. Cançado, C.B., 1993. An experimental and clinical assay with ketoconazole in the treatment of Chagas disease. Mem. Inst. Oswaldo Cruz 88, 149.

Britto, C.C., 2009. Usefulness of PCR-based assays to assess drug efficacy in Chagas disease chemotherapy: value and limitations. Mem. Inst. Oswaldo Cruz 104, 122.

Caballero, Z.C., Sousa, O.E., Marques, W.P., Saez-Alquezar, A., Umezawa, E.S. 2007. Evaluation of serological tests to identify Trypanosomacruzi infection in humans and determine cross-reactivity with Trypanosoma rangeli and Leishmania spp. Clin. Vaccine Immunol. 14, 1045.

Camargo, E.P. 1964. Growth and differentiation in Trypanosoma cruzi. I. Origin of metacyclic trypanosomes in liquid media. Rev. Inst. Med. Trop. Sao Paulo 6, 6.

Cançado, J.R., 2002. Long term evaluation of etiological treatment of chagas disease with benznidazole. Rev. Inst. Med. Trop. Sao Paulo 44, 29

Cerisola, J.A., Alvarez, M., Bock, M., Wegner, D.A., 1971. Comparison of a new antigen from amastigotes of Trypanosoma cruzi and an antigen from epimastigotes for the diagnosis of Chagas disease by the indirect immunofluorescence test. Rev. Inst. Med. Trop. 13, 162

Cerisola, J.A., Rohweddwe, R., Segura, E.L., Del Prado, C.E.,Alvarez, M., Martini, G.J.W., 1974. El xenodiagnóstico. Buenos Aires: Imp. Inst. Nac. Invest. Cardiovasc. p. 157.

Chagas, C., 1909. Nova Tripanozomiase Humana. Estudo sobre a morfologia e o ciclo evolutivo do Schizotrypanum cruzi. n. gen., n. sp. agente etiológico de nova entidade mórbida do homem. Mem. Inst. Oswaldo Cruz 2, 159.

Chiari, E., Dias, J.C.P., Lana, M., Chiari, C.A., 1989. Hemocultures for the parasitological diagnosis of human chronic Chaga's disease. Rev. Soc Bras. Med. Trop. 22, 19.

Consenso Brasileiro em Doença de Chagas, 2005. Ministério da Saúde, Secretaria de Vigilância em Saúde. http://portal.saude.gov.br/portal/ arquivos/pdf/consenso_chagas.pdf.

Cordeiro, F.D., Martins-Filho, A.O., Da Costa Rocha, M.O., Adad, S.J., CorreaOliveira, R., Romanha, A.J., 2001. Anti-Trypanosoma cruzi immunoglobulin
G1 can be a useful tool for diagnosis and prognosis of human Chagas disease. Clin. Diagn. Lab. Immunol. 8, 112.

Coura, J.R.,Castro, S.L., 2002. A critical review on Chagas disease chemotherapy. Mem. Inst. Oswaldo Cruz 97, 3.

Dias, J.C.P., 1995. Natural history of Chagas' disease. Arq. Bras. Cardiol. 65, 359.

Dias, J.C.P., 2000. Epidemiological surveillance of Chagas disease. Cad. Saude Publica 16, 43.

Fabbro, D., Arias, E., Streiger, M., Piacenza, M., Ingarano, M., Del Barco, M., Amicone, N., 2000. Evolutive behavior towards cardiomyopathy of treated (nifurtimoxor benznidazole) and untreated chronic chagasic patients. Rev. Inst. Med. Trop. Sao Paulo 42, 99.

Flores-Chávez, M.,Cruz, I., Rodríguez, M.,Nieto, J., Franco, E., Gárate, T.,Cañavate, C., 2010. Comparison of conventional and non-conventional serological tests for the diagnosis of imported Chagas disease in Spain. Enferm. Infecc. Microbiol. Clin. 28, 284.

Flores-García, Y., Rosales-Encina, J.L., Satoskar, A.R., Talamás-Rohana, P., 2011. IL-10-IFN- $\gamma$ double producers CD4 $+\mathrm{T}$ cells are induced by immunization with an amastigote stage specific derived recombinant protein of Trypanosoma cruzi. Int. J. Biol. Sci. 7, 1093.

Gadelha, A.A., Verçosa, A.F., Lorena, V.M., Nakazawa, M.,Carvalho, A.B.,Souza, W.V., Ferreira, A.G., Silva, E.D., Krieger, M.A., Goldenberg, S., Gomes, Y.M., 2003. Chagas' disease diagnosis: comparative analysis of recombinant ELISA with conventional ELISA and the haemagglutination test. Vox Sang. 85, 165.

Galvão, L.M., Nunes, R.M.B., Cançado, J.R., Brener, Z., Krettli, A.U., 1993. Litic antibody titre as a means of assessing cure after treatment of Chagas disease: a ten years follow-up study. Trans. R. Soc. Trop. Med. Hyg. 87, 220.

Gomes, M.L., Macedo, A.M., Vago, A.R., Pena, S.D., Galvão, L.M., Chiari, E., 1998. Trypanosoma cruzi: optimization of polymerase chain reaction for detection in human blood. Exp. Parasitol. 88, 28.

Gomes, Y.M.,Pereira, V.R.,Nakazawa, M.,Rosa, D.S.,Barros, M.D.,Ferreira, A.G.,Silva, E.D., Ogatta, S.F., Krieger, M.A., Goldenberg, S., 2001. Serodiagnosis of chronic Chagas infection by using EIE-Recombinant-Chagas-Biomanguinhos kit. Mem. Inst. Oswaldo Cruz 96, 497.

Gomes, Y.M., Lorena, V.M., Luquetti, A.O., 2009. Diagnosis of Chagas disease: what has been achieved? What remains to be done with regard to diagnosis and follow up studies? Mem. Inst. Oswaldo Cruz 104, 115

Krettli, A.U., Brener, Z., 1976. Protective effects of specific antibodies in Trypanosoma cruzi infections. J. Immunol. 116, 755.

Krettli, A.U.,Brener, Z., 1982. Resistance against Trypanosoma cruzi associated to anti-living trypomastigote antibodies. J. Immunol. 128, 2009.

Krettli, A.U., Cançado, J.R., Brener, Z., 1982. Effect of specific chemotherapy on the levels of lytic antibodies in Chagas disease. Trans. R. Soc. Trop. Med. Hyg. 76, 334.

Lana, M., Martins-Filho, O.A., 2009. Serological methods as a tool to monitor post-therapeutic cure in Chagas disease. Rev. Soc. Bras. Med. Trop. 42, 54.

Lana, M., Lopes, L.A., Martins, H.R., Bahia, M.T., Machado-De-Assis, G.F. Wendling, A.P., Martins-Filho, O.A., Montoya, R.A., Dias, J.C.P., AlbajarViñas, P., Coura, J.R., 2009. Clinical and laboratorial status of patients with chronic Chagas disease living in a vector-controlled area in Minas Gerais, Brazil, before and nine years after a etiological treatment. Mem. Inst. Oswaldo Cruz 104, 1139.

Luquetti, A.O., Rassi, A., 2000. Diagnóstico laboratorial da infecção pelo Trypanosoma cruzi, In: Brener, Z., Andrade, Z., Barral-Neto, M. (Eds.), Trypanosoma cruzi e doença de Chagas, 2nd ed.Guanabara Koogan, Rio de Janeiro, Brazil, p. 344.

Machado-De-Assis, G.F.,Silva, A.R., Do Bem, V.A., Bahia, M.T., Martins-Filho, O.A., Dias, J.C., Albajar-Viñas, P., Torress, R.M., Lana, M., 2012. Post-therapeutic cure criteria in Chagas' disease: conventional serology followed by supplementary serological, parasitological, and molecular tests. Clin. Vaccine Immunol. 19, 1283

Martins-Filho, O.A.,Pereira, M.E.S.,Carvalho, J.A.C., Cançado, J.R., Brener, Z., 1995. Flow cytometry, a new approach to detect anti-live trypomastigote antibodies and monitor the efficacy of specific treatment in human chagas disease. Clin. Diagn. Lab. Immunol. 2, 569.

Martins-Filho, O.A., Eloi-Santos, S.M., Carvalho, A.T., Oliveira, R.C., Rassi, A., Luquetti, A.O., Rassi, G.G., Brener, Z., 2002. Double-blind study to evaluate flow cytometry analysis of anti-live trypomastigote antibodies for monitoring treatment efficacy in cases of human Chagas' disease. Clin. Diagn. Lab. Immunol. 9, 1107.

Matos, C.S., Coelho-Dos-Reis, J.G., Rassi, A., Luquetti, A.O., Dias, J.C.P.,Eloi-Santos, S.M., Gomes, I.T., Vitelli-Avelar, D.M., Wendling, A.P.B., Rocha, R.D.R., Teixeira-Carvalho, A., Peruhype-Magalhães, V., Andrade, M.C., MartinsFilho, A.O., 2011. Applicability of an optimized non-conventional flow cytometry method to detect anti-Trypanosoma cruzi immunoglobulin $\mathrm{G}$ for the serological diagnosis and cure assessment following chemotherapeutic treatment of Chagas disease. J. Immunol. Methods 369, 22.

Matsumoto, T.K., Hoshino-Shimizu, S., Nakamura, P.M., Andrade Jr., H.F., Umezawa, E.S., 1993. High resolution of Trypanosomacruziamastigote antigen in serodiagnosis of different clinical forms of Chagas' disease. J. Clin. Microbiol. 31, 1486. 
Moncayo, A., Silveira, A.C., 2009. Current epidemiological trends for Chagas disease in Latin America and future challenges in epidemiology, surveillance and health policy. Mem. Inst. Oswaldo Cruz 104, 17.

Nakazawa, M., Rosa, D.S., Pereira, V.R., Moura, M.O., Furtado, V.C., Souza, W.V., Barros, M.N., Abath, F.G., Gomes, Y.M., 2001. Excretory-secretory antigens of Trypanosoma cruzi are potentially useful for serodiagnosis of chronic Chagas' disease. Clin. Diagn. Lab. Immunol. 28, 1024.

Oelemann, W.M.R.,Teixeira, M.G.M., Veríssimo Da Costa, G.C., Borges-Pereira, J., De Castro, J.A.F., Coura, J.R., Peralta, J.M., 1998. Evaluation of three commercial enzyme-linked immunosorbent assays for diagnosis of Chagas disease. J. Clin. Microbiol. 36, 2423.

Pereira, J.B., Junqueira, A.C.V.,Santos, L.C., Castro, J.A.F., Araujo, I.B., Coura, J.R., 1996. Xenodiagnóstico na doença de Chagas crônica. I. Sensibilidade de Panstrongylus megistus e Triatoma infestans. Rev. Soc. Bras. Med. Trop. 29, 241

Portela-Lindoso, A.A., Shikanai-Yasuda, M.A., 2003. Chronic Chagas' disease: from xenodiagnosis and hemoculture to polymerase chain reaction. Rev. Saude Publica 37, 107.

Primavera, K.S.C. Umezawa, E.S.,Peres, B.A. Camargo, M.E.,Hoshino-Shimizu, S. 1990. Chagas' disease: IgA, IgM and IgG antibodies to T. cruzi amastigote, tripomastigote and epimastigote antigens in acute and in different chronic forms of the disease. Rev. Inst. Med. Trop. 32, 172

Ramos-Ligonio, A., López-Monteon, A., Talamás-Rohana, P., Rosales-Encina, J.L., 2004. Recombinant Ssp-4 protein from Trypanosomacruziamastigotes regulates nitric oxide production by macrophages. Parasite Immunol. 26, 409

Rassi, A.J.R., Rassi, A., Marin-Neto, J., 2010. Chagas disease. Lancet 375, 1388.

Sánchez, N.O.,Sánchez, V.F.J.,Lacunza, C.D.,García, B.M.F.,Mora, M.C.,Uncos, A.D., Basombrío, M.A., 2008. Serological evaluation of specific-antibody levels in patients treated for chronic Chagas' disease. Clin. Vaccine Immunol. 15, 297.

Schenone, H., Rojas, A., Castillo, D., 2000. Comparative study of sensitivity and mortality of Triatoma infestan nymphs III and IV used in the xenodiagnosis of chronic chagasic patients. Bol. Chil. Parasitol. 55, 14
Schmunis, G.A., 2007. Epidemiology of Chagas disease in non-endemic countries: the role of international migration. Mem. Inst. Oswaldo Cruz $102,75$.

Swets, J.A., 1988. Measuring the accuracy of diagnostic systems. Science 240, 1285.

Umezawa, E.S., Nascimento, M.S., Stolf, A.M., 2001. Enzyme-linked immunosorbent assay with Trypanosoma cruzi excreted-secreted antigens (TESAELISA) for serodiagnosis of acute and chronic Chagas' disease. Diagn. Microbiol. Infect. Dis. 39, 169.

Vexenat, A.C., Santna, J.M., Teixeira, A.R., 1996. Cross-reactivity of antibodies in human infections by the kinetoplastid protozoa Trypanosoma cruzi, Leishmania chagasi and Leishmania (viannia) braziliensis. Rev. Inst. Med. Trop. Sao Paulo 38, 177.

Viotti, R., Vigliano, C.,Armenti, H.,Segura, E., 1994. Treatment of chronic Chagas disease with benznidazole: clinical and serologic evolution of patients with long-term follow-up. Am. Heart J. 127, 151.

Vitelli-Avelar, D., Sathler-Avelar, R., Wendling, A.P.B., Rocha, R.D.R., TeixeiraCarvalho, A., Martins, N.E., Dias, J.C.P., Rassi, A., Luquetti, A.O., Elói-Santos, S.M., Martins-Filho, A.O., 2007. Non-conventional flow cytometry approaches to detect anti-Trypanosoma cruzi immunoglobulin $\mathrm{G}$ in the clinical laboratory. J. Immunol. Methods 318, 102.

Wendling, A.P., Vitelli-Avelar, D.M., Sathler-Avelar, R., Geiger, S.M., TeixeiraCarvalho, A., Gontijo, E.D., Eloi-Santos, S.M., Martins-Filho, O.A., 2011. The use of IgG antibodies in conventional and non-conventional immunodiagnostic tests for early prognosis after treatment of Chagas disease. J. Immunol. Methods 370, 24

World Health Organization, 2010. First WHO report on neglected tropical diseases. Working to Overcome the Global Impact of Neglected Tropical Diseases (http://www.who.int/neglected_diseases/2010report/en/). 